\title{
A Combinatorial Proof of a Symmetric $q$-Pfaff-Saalschütz Identity
}

\author{
Victor J. W. Guo \\ Center for Combinatorics, LPMC \\ Nankai University, Tianjin 300071, People's Republic of China \\ jwguo@eyou.com \\ Jiang Zeng \\ Institut Girard Desargues, Université Claude Bernard (Lyon I) \\ F-69622 Villeurbanne Cedex, France \\ zeng@igd.univ-lyon 1 .fr
}

Submitted: Oct 15, 2004; Accepted: Jan 27, 2005; Published: Feb 4, 2005

Mathematics Subject Classifications: 05A17, 33D15

\begin{abstract}
We give a bijective proof of a symmetric $q$-identity on ${ }_{4} \phi_{3}$ series, which is a symmetric generalization of the famous $q$-Pfaff-Saalschütz identity. An elementary proof of this identity is also given.
\end{abstract}

\section{Introduction}

Throughout this paper we regard $q$ as an indeterminate, and we follow the notation and terminology in [5]. The q-shifted factorials are defined by

$$
\left(a_{1}, a_{2}, \ldots, a_{m} ; q\right)_{n}=\left(a_{1} ; q\right)_{n}\left(a_{2} ; q\right)_{n} \cdots\left(a_{m} ; q\right)_{n}
$$

where $(a ; q)_{0}=1$ and $(a ; q)_{n}=\prod_{k=0}^{n-1}\left(1-a q^{k}\right)$ for $n \geq 1$.

In 1990, the second author [11] obtained a symmetric extension of a formula due to Ramanujan-Bailey, of which the analytical proof led to the following $q$-identity:

$$
\begin{aligned}
& \frac{(x z, y z ; q)_{m}(z ; q)_{n}}{(q, x y z ; q)_{m}(q ; q)_{n}}{ }_{4} \phi_{3}\left[\begin{array}{l}
q^{-n}, x, y, v q^{m} \\
v, x y z q^{m}, q^{1-n} / z,
\end{array} ; q, q\right] \\
& =\frac{(x z, y z ; q)_{n}(z ; q)_{m}}{(q, x y z ; q)_{n}(q ; q)_{m}}{ }_{4} \phi_{3}\left[\begin{array}{l}
q^{-m}, x, y, v q^{n} \\
v, x y z q^{n}, q^{1-m} / z,
\end{array} ; q, q\right]
\end{aligned}
$$


where $m, n \in \mathbb{N}$. Indeed, applying Sears' transformation [5, p. 360, (III.15)] with $a=$ $x, b=y, c=v q^{m}, d=v, e=x y z q^{m}$, and $f=q^{1-n} / z$ to the left-hand side of (1.1) yields the following identity

$$
\begin{aligned}
& \frac{(x z, y z ; q)_{m}(z ; q)_{n}}{(q, x y z ; q)_{m}(q ; q)_{n}}{ }_{4} \phi_{3}\left[\begin{array}{l}
q^{-n}, x, y, v q^{m} \\
v, x y z q^{m}, q^{1-n} / z,
\end{array} ; q, q\right] \\
& \quad=\frac{(x z ; q)_{m}(x z ; q)_{n}(y z ; q)_{m+n}}{(x y z ; q)_{m+n}(q ; q)_{m}(q ; q)_{n}}{ }_{1} \phi_{3}\left[\begin{array}{l}
q^{-n}, x, v / y, q^{-m} \\
v, x z, q^{1-m-n} / y z,
\end{array} ; q, q\right] .
\end{aligned}
$$

It follows that the left-hand side of (1.1) is symmetric in $m$ and $n$, which is exactly what (1.1) means.

In order to give a combinatorial proof of (1.1), we first rewrite (1.1) as

$$
\begin{aligned}
& \frac{(x z, y z ; q)_{m}}{(q, x y z ; q)_{m}} \sum_{k=0}^{n} \frac{\left(x, y, v q^{m} ; q\right)_{k}(z ; q)_{n-k}}{\left(q, v, x y z q^{m} ; q\right)_{k}(q ; q)_{n-k}} z^{k} \\
& \quad=\frac{(x z, y z ; q)_{n}}{(q, x y z ; q)_{n}} \sum_{k=0}^{m} \frac{\left(x, y, v q^{n} ; q\right)_{k}(z ; q)_{m-k}}{\left(q, v, x y z q^{n} ; q\right)_{k}(q ; q)_{m-k}} z^{k} .
\end{aligned}
$$

Letting $x=q^{-(a-r)}, y=q^{-(b-r)}, z=q^{a+b+1}, v=q^{-(e-r)}, n=c-r$, and $m=d-r$, and using the formulas

$$
\left(q^{-N} ; q\right)_{k}=(-1)^{k} q^{-k N+\left(\begin{array}{c}
k \\
2
\end{array}\right)} \frac{(q ; q)_{N}}{(q ; q)_{N-k}}, \quad\left(q^{N+1} ; q\right)_{k}=\frac{(q ; q)_{N+k}}{(q ; q)_{N}}, \quad N \in \mathbb{N},
$$

the left-hand side of (1.3) becomes

$$
\begin{aligned}
& \frac{\left(q^{b+r+1}, q^{a+r+1} ; q\right)_{d-r}}{\left(q, q^{2 r+1} ; q\right)_{d-r}} \sum_{k=0}^{c-r} \frac{\left(q^{-(a-r)}, q^{-(b-r)}, q^{-(e-d)} ; q\right)_{k}\left(q^{a+b+1} ; q\right)_{c-r-k}}{\left(q, q^{-(e-r)}, q^{d+r+1} ; q\right)_{k}(q ; q)_{c-r-k}} q^{(a+b+1) k} \\
& =\frac{(q ; q)_{b+d}(q ; q)_{a+d}(q ; q)_{2 r}(q ; q)_{a-r}(q ; q)_{b-r}(q ; q)_{e-d}}{(q ; q)_{b+r}(q ; q)_{a+r}(q ; q)_{d-r}(q ; q)_{a+b}(q ; q)_{e-r}} \\
& \quad \times \sum_{k=0}^{c-r} q^{k(k+d+r)} \frac{(q ; q)_{a+b+c-r-k}(q ; q)_{e-r-k}}{(q ; q)_{k}(q ; q)_{a-r-k}(q ; q)_{b-r-k}(q ; q)_{e-d-k}(q ; q)_{d+r+k}(q ; q)_{c-r-k}}
\end{aligned}
$$

where $a, b, c, d, e$ are nonnegative integers and $r$ is an integer such that $r \leq \min \{a, b, c, d\}$ and $e \geq \max \{c, d\}$.

Exchanging $c$ and $d$, we obtain a similar expression for the right-hand side of (1.3). Hence, after simplification, we see that (1.3) is equivalent to

$$
\begin{aligned}
& \sum_{k=0}^{c-r} \frac{q^{k(k+d+r)}(q ; q)_{b+d}(q ; q)_{a+d}(q ; q)_{e-d}(q ; q)_{a+b+c-r-k}(q ; q)_{e-r-k}}{(q ; q)_{d-r}(q ; q)_{k}(q ; q)_{a-r-k}(q ; q)_{b-r-k}(q ; q)_{e-d-k}(q ; q)_{d+r+k}(q ; q)_{c-r-k}} \\
& \quad=\sum_{k=0}^{d-r} \frac{q^{k(k+c+r)}(q ; q)_{b+c}(q ; q)_{a+c}(q ; q)_{e-c}(q ; q)_{a+b+d-r-k}(q ; q)_{e-r-k}}{(q ; q)_{c-r}(q ; q)_{k}(q ; q)_{a-r-k}(q ; q)_{b-r-k}(q ; q)_{e-c-k}(q ; q)_{c+r+k}(q ; q)_{d-r-k}}
\end{aligned}
$$


Finally, shifting $k$ to $k-r$ and using the $q$-binomial coefficient

$$
\left[\begin{array}{c}
M \\
N
\end{array}\right]=\left[\begin{array}{c}
M \\
N
\end{array}\right]_{q}= \begin{cases}\frac{(q ; q)_{M}}{(q ; q)_{N}(q ; q)_{M-N}}, & \text { if } 0 \leq N \leq M \\
0, & \text { otherwise }\end{cases}
$$

we can rewrite (1.4) in the following form:

$$
\begin{gathered}
\sum_{k \in \mathbb{Z}} q^{(k-r)(k+d)}\left[\begin{array}{c}
a+b+c-k \\
a-k
\end{array}\right]\left[\begin{array}{l}
b+d \\
b-k
\end{array}\right]\left[\begin{array}{l}
c-r \\
c-k
\end{array}\right]\left[\begin{array}{l}
e-k \\
d-r
\end{array}\right] \\
=\sum_{k \in \mathbb{Z}} q^{(k-r)(k+c)}\left[\begin{array}{c}
a+b+d-k \\
b-k
\end{array}\right]\left[\begin{array}{l}
a+c \\
a-k
\end{array}\right]\left[\begin{array}{l}
e-c \\
k-r
\end{array}\right]\left[\begin{array}{l}
e-k \\
d-k
\end{array}\right] .
\end{gathered}
$$

Note that setting $d=r$ and letting $e \longrightarrow+\infty$ in (1.5) we recover the $q$-Pfaff-Saalschütz identity:

$$
\sum_{k \in \mathbb{Z}} \frac{q^{k^{2}-r^{2}}[a+b+c-k] !}{[a-k] ![b-k] ![c-k] ![k-r] ![k+r] !}=\left[\begin{array}{l}
a+b \\
a+r
\end{array}\right]\left[\begin{array}{l}
a+c \\
c+r
\end{array}\right]\left[\begin{array}{l}
b+c \\
b+r
\end{array}\right],
$$

where $[n] !=(q ; q)_{n} /(1-q)^{n}$ for $n \geq 0$ and $1 /[n] !=0$ for $n<0$. In the 1980's several authors $[3,6,12]$ published combinatorial proofs of the $q$-Pfaff-Saalschütz identity. The main object of this paper is to provide a bijective proof of (1.5) by generalizing Zeilberger's combinatorial proof of the $q$-Pfaff-Saalschütz identity (1.6).

On the other hand, setting $x=0$ and letting $v \longrightarrow \infty$, identity (1.3) reduces to:

$$
\frac{(y z ; q)_{m}}{(q ; q)_{m}} \sum_{k=0}^{n} \frac{(y ; q)_{k}(z ; q)_{n-k}}{(q ; q)_{k}(q ; q)_{n-k}} q^{m k} z^{k}=\frac{(y z ; q)_{n}}{(q ; q)_{n}} \sum_{k=0}^{m} \frac{(y ; q)_{k}(z ; q)_{m-k}}{(q ; q)_{k}(q ; q)_{m-k}} q^{n k} z^{k}
$$

while (1.2) reduces to

$$
{ }_{2} \phi_{1}\left[\begin{array}{l}
q^{-n}, y \\
q^{1-n} / z, ; q, q^{m+1}
\end{array}\right]=\frac{(y z ; q)_{m+n}}{(y z ; q)_{m}(z ; q)_{n}}{ }_{2} \phi_{1}\left[\begin{array}{l}
q^{-m}, q^{-n} \\
q^{1-m-n} / y z,
\end{array} ; q, q / y\right],
$$

or

$$
\sum_{k=0}^{n} \frac{(y ; q)_{k}(z ; q)_{n-k}}{(q ; q)_{k}(q ; q)_{n-k}} q^{m k} z^{k}=\frac{(q ; q)_{m}}{(y z ; q)_{m}} \sum_{k=0}^{\min \{m, n\}} \frac{(y z ; q)_{m+n-k} q^{\left(\begin{array}{c}
k \\
2
\end{array}\right)}(-z)^{k}}{(q ; q)_{k}(q ; q)_{m-k}(q ; q)_{n-k}} .
$$

The first author [7] has recently proved the $y=q / z$ case of (1.7) by using combinatorics of partition theory. Hence it is natural to ask for a combinatorial proof of (1.7) by extending the argument of [7]. Note that (1.8) shows that the left-hand side of (1.7) is symmetric in $m$ and $n$, which establishes (1.7).

This paper is organized as follows: in Section 2 we give the bijective proof of (1.5) in the framework of words, and in Section 3 the combinatorial proof of (1.8) using partitions of integers. Finally, in Section 4, we give a short proof of (1.3) from scratch, in the same vein as the first author's approach to some other well-known $q$-identities [8]. 


\section{A Bijective Proof of Equation (1.5)}

Let $M\left(1^{n_{1}}, \ldots, m^{n_{m}}\right)$ denote the set of rearrangements of the word $1^{n_{1}} 2^{n_{2}} \ldots m^{n_{m}}$. An inversion in a word $w=w_{1} w_{2} \cdots w_{n}$ on the alphabet $\{1, \ldots, m\}$ is a pair of indices $(i, j)$ such that $i<j$ and $w_{i}>w_{j}$. The number of inversions of $w$ is denoted by $\operatorname{inv}(w)$. For instance, for $w=131223211 \in M\left(1^{4}, 2^{3}, 3^{2}\right)$, we have $\operatorname{inv}(w)=15$. It is folklore $[2$, Theorem 3.6] that

$$
\sum_{w \in M\left(1^{n_{1}}, \ldots, m^{n_{m}}\right)} q^{\operatorname{inv}(w)}=\frac{\left[n_{1}+\cdots+n_{m}\right] !}{\left[n_{1}\right] ! \cdots\left[n_{m}\right] !} .
$$

On the other hand, for any word $w$ on the alphabet of two letters $\{a, b\}$ with $a<b$, we define $\imath_{a b}(w)$ to be the word obtained from $w$ by reversing the order of letters of $w$ and then interchanging the letters $a$ 's and $b$ 's. For instance, if $a=1$ and $b=2$, then $\imath_{12}(11222221)=21111122$. It is easy to see that $\imath_{a b}$ is an involution such that $\operatorname{inv}(w)=\operatorname{inv}\left(\imath_{a b}(w)\right)$.

If $w_{1}$ and $w_{2}$ are two words, we denote by $w_{1} w_{2}$ their concatenation.

Lemma 2.1 Let $b, c, d, e \in \mathbb{N}$ and $r \in \mathbb{Z}$ such that $r \leq \min \{b, c, d, e, d+e-c\}$. For any $k \in \mathbb{Z}$, define the sets

$$
\begin{aligned}
& A_{k}=M\left(2^{b-k}, 3^{k+d}\right) \times M\left(2^{k-r}, 4^{c-k}\right) \times M\left(3^{e-k}, 4^{d-r}\right), \\
& B_{k}=M\left(2^{b-k}, 3^{k+c}\right) \times M\left(2^{k-r}, 4^{e+d-c-k}\right) \times M\left(3^{d-k}, 4^{e-r}\right),
\end{aligned}
$$

where $M\left(a^{i}, b^{j}\right)=\emptyset$ if $i<0$ or $j<0$ by convention. Set $A=\cup_{k} A_{k}$ and $B=\cup_{k} B_{k}$. For each triple $w=\left(w_{1}, w_{2}, w_{3}\right) \in A \cup B$, define the statistic $\operatorname{inv}(w)=\operatorname{inv}\left(w_{1} w_{2}\right)+\operatorname{inv}\left(w_{3}\right)$. Then there is a bijection $\theta: A \longrightarrow B$ such that $\operatorname{inv}(w)=\operatorname{inv}(\theta(w))$.

Proof. Start with a triple $\left(w_{1}, w_{2}, w_{3}\right) \in A_{k}$. Replacing all the 4 's in $w_{2}$ by the leftmost $c-k$ letters in $w_{3}$ one by one, we obtain a word $w_{2}^{\prime}$. Denote by $w_{2}^{\prime \prime}$ the word obtained from $w_{2}^{\prime}$ by deleting all the 4's. Let $v_{3}^{\prime}$ be the word obtained from $w_{2}^{\prime}$ by replacing every 2 by 3 . Note that both $w_{1}$ and $w_{2}^{\prime \prime}$ are words on $\{2,3\}$. Let $v_{1}$ be the word corresponding to the leftmost $b+c$ letters in $w_{1} w_{2}^{\prime \prime}$, and let $v_{1}^{\prime}$ be the word such that $v_{1} v_{1}^{\prime}=w_{1} w_{2}^{\prime \prime}$.

Let $w_{3}^{\prime}$ be the word obtained from $w_{3}$ by deleting the leftmost $c-k$ letters. It is easy to see that the number of 4 's in $w_{3}^{\prime}$ is exactly equal to the length of $v_{1}^{\prime}$. Let $w_{3}^{\prime \prime}=\imath_{34}\left(w_{3}^{\prime}\right)$. Replacing all the letters 3 's in $w_{3}^{\prime \prime}$ by those in $v_{1}^{\prime}$ one by one, we obtain a word $w_{3}^{\prime \prime \prime}$. Let $v_{2}$ be the word obtained from $w_{3}^{\prime \prime \prime}$ by replacing every 3 by 4 , and let $v_{2}^{\prime}$ be the word obtained from $w_{3}^{\prime \prime \prime}$ by deleting all the 2's. Finally, let $v_{3}=v_{2}^{\prime} \imath_{34}\left(v_{3}^{\prime}\right)$. Clearly $v=\left(v_{1}, v_{2}, v_{3}\right) \in B$.

Conversely, if $v=\left(v_{1}, v_{2}, v_{3}\right) \in B_{k}$, then the above procedure with $c-k$ changed to $e+d-c-k$ and $b+c$ to $b+d$ also defines a mapping $\theta$ from $B$ to $A$ such that $\theta^{2}$ is the identity mapping. Thus, the mapping $\theta: w \mapsto v$ is a bijection from $A$ to $B$.

It remains to show that $\operatorname{inv}(w)=\operatorname{inv}(v)$. Let $|u|_{i}$ denote the number of occurrences of $i$ in the word $u$. Note that, in our construction from $w$ to $v$, we have the following 
obvious relations:

$$
\begin{aligned}
\operatorname{inv}\left(w_{2}\right) & =\operatorname{inv}\left(w_{2}^{\prime}\right)=\operatorname{inv}\left(w_{2}^{\prime \prime}\right)+\operatorname{inv}\left(v_{3}^{\prime}\right), \\
\operatorname{inv}\left(w_{3}^{\prime \prime \prime}\right) & =\operatorname{inv}\left(w_{3}^{\prime \prime}\right)+\operatorname{inv}\left(v_{1}^{\prime}\right)=\operatorname{inv}\left(w_{3}^{\prime}\right)+\operatorname{inv}\left(v_{1}^{\prime}\right), \\
\operatorname{inv}\left(w_{3}^{\prime \prime \prime}\right) & =\operatorname{inv}\left(v_{2}\right)+\operatorname{inv}\left(v_{2}^{\prime}\right), \\
\operatorname{inv}\left(v_{3}\right) & =\operatorname{inv}\left(v_{2}^{\prime}\right)+\operatorname{inv}\left(v_{3}^{\prime}\right)+\left|v_{2}^{\prime}\right|_{4} \cdot\left|v_{3}^{\prime}\right|_{4}, \\
\operatorname{inv}\left(v_{1}\right)+\operatorname{inv}\left(v_{1}^{\prime}\right)+\left|v_{1}\right|_{3} \cdot\left|v_{1}^{\prime}\right|_{2} & =\operatorname{inv}\left(w_{1}\right)+\operatorname{inv}\left(w_{2}^{\prime \prime}\right)+\left|w_{1}\right|_{3} \cdot\left|w_{2}^{\prime \prime}\right|_{2}, \\
& =\operatorname{inv}\left(w_{1}\right)+\operatorname{inv}\left(w_{2}^{\prime \prime}\right)+\left|w_{1}\right|_{3} \cdot\left|w_{2}\right|_{2}, \\
\left|v_{2}\right|_{2} & =\left|v_{1}^{\prime}\right|_{2}, \\
\left|v_{2}^{\prime}\right|_{4} & =\left|w_{3}^{\prime \prime \prime}\right|_{4}=\left|w_{3}^{\prime \prime}\right|_{4}=\left|w_{3}^{\prime}\right|_{3}, \\
\left|v_{3}^{\prime}\right|_{4} & =\left|w_{2}^{\prime}\right|_{4}, \\
\operatorname{inv}\left(w_{3}\right) & =\operatorname{inv}\left(w_{3}^{\prime}\right)+\left|w_{3}^{\prime}\right|_{3} \cdot\left|w_{2}^{\prime}\right|_{4} .
\end{aligned}
$$

It follows that

$$
\begin{aligned}
& \operatorname{inv}\left(v_{1} v_{2}\right)+\operatorname{inv}\left(v_{3}\right) \\
& \quad=\operatorname{inv}\left(v_{1}\right)+\operatorname{inv}\left(v_{2}\right)+\left|v_{1}\right|_{3} \cdot\left|v_{2}\right|_{2}+\operatorname{inv}\left(v_{2}^{\prime}\right)+\operatorname{inv}\left(v_{3}^{\prime}\right)+\left|v_{2}^{\prime}\right|_{4} \cdot\left|v_{3}^{\prime}\right|_{4} \\
& \quad=\operatorname{inv}\left(v_{1}\right)+\operatorname{inv}\left(v_{1}^{\prime}\right)+\operatorname{inv}\left(w_{3}^{\prime}\right)+\left|v_{1}\right|_{3} \cdot\left|v_{2}\right|_{2}+\operatorname{inv}\left(v_{3}^{\prime}\right)+\left|w_{3}^{\prime}\right|_{3} \cdot\left|w_{2}^{\prime}\right|_{4} \\
& \quad=\operatorname{inv}\left(w_{1}\right)+\operatorname{inv}\left(w_{2}^{\prime \prime}\right)+\left|w_{1}\right|_{3} \cdot\left|w_{2}\right|_{2}+\operatorname{inv}\left(w_{3}^{\prime}\right)+\operatorname{inv}\left(v_{3}^{\prime}\right)+\left|w_{3}^{\prime}\right|_{3} \cdot\left|w_{2}^{\prime}\right|_{4} \\
& \quad=\operatorname{inv}\left(w_{1}\right)+\operatorname{inv}\left(w_{2}\right)+\left|w_{1}\right|_{3} \cdot\left|w_{2}\right|_{2}+\operatorname{inv}\left(w_{3}^{\prime}\right)+\left|w_{3}^{\prime}\right|_{3} \cdot\left|w_{2}^{\prime}\right|_{4} \\
& \quad=\operatorname{inv}\left(w_{1} w_{2}\right)+\operatorname{inv}\left(w_{3}\right) .
\end{aligned}
$$

This completes the proof.

Example 2.1 Let $b=7, c=8, d=9, e=10, r=0$ and $k=4$. If

$$
w=\left(w_{1}, w_{2}, w_{3}\right)=(3323333233323333,42244242,334344344434344),
$$

then, $w_{2}^{\prime}=32234232$, i.e., $w_{2}^{\prime \prime}=3223232$ and $v_{3}^{\prime}=33334333$. So, $v_{1}=332333323332333$ and $v_{1}^{\prime}=33223232$.

On the other hand, $w_{3}^{\prime}=44344434344$, i.e., $w_{3}^{\prime \prime}=33434333433$. Hence, $w_{3}^{\prime \prime \prime}=$ 33424232432 , i.e., $v_{2}=44424242442$ and $v_{2}^{\prime}=3344343$. Thus, $v_{3}=v_{2}^{\prime} \imath_{34}\left(v_{3}^{\prime}\right)=$ 334434344434444. Namely,

$$
v=\left(v_{1}, v_{2}, v_{3}\right)=(332333323332333,44424242442,334434344434444) .
$$

It is easy to see that $\operatorname{inv}(w)=\operatorname{inv}(v)=95$.

Proof of (1.5). Let $M=\cup_{k} M_{k}$ and $N=\cup_{k} N_{k}$, where

$$
\begin{aligned}
M_{k} & =M\left(1^{b+c}, 2^{a-k}\right) \times M\left(1^{b-k}, 3^{k+d}\right) \times M\left(2^{k-r}, 4^{c-k}\right) \times M\left(3^{e-k}, 4^{d-r}\right), \\
N_{k} & =M\left(1^{a+d}, 2^{b-k}\right) \times M\left(1^{a-k}, 3^{k+c}\right) \times M\left(2^{k-r}, 4^{e+d-c-k}\right) \times M\left(3^{d-k}, 4^{e-r}\right) .
\end{aligned}
$$


For each element $w=\left(w_{1}, w_{2}, w_{3}, w_{4}\right)$ of $M \cup N$ define

$$
\operatorname{inv}(w)=\operatorname{inv}\left(w_{1}\right)+\operatorname{inv}\left(w_{2} w_{3}\right)+\operatorname{inv}\left(w_{4}\right) .
$$

Then, in view of $(2.1)$, we have

$$
\begin{aligned}
& \sum_{w \in M_{k}} q^{\operatorname{inv}(w)}=q^{(k-r)(k+d)}\left[\begin{array}{c}
a+b+c-k \\
a-k
\end{array}\right]\left[\begin{array}{l}
b+d \\
b-k
\end{array}\right]\left[\begin{array}{c}
c-r \\
c-k
\end{array}\right]\left[\begin{array}{c}
e+d-r-k \\
d-r
\end{array}\right], \\
& \sum_{w \in N_{k}} q^{\operatorname{inv}(w)}=q^{(k-r)(k+c)}\left[\begin{array}{c}
a+b+d-k \\
b-k
\end{array}\right]\left[\begin{array}{c}
a+c \\
a-k
\end{array}\right]\left[\begin{array}{c}
e+d-r-c \\
k-r
\end{array}\right]\left[\begin{array}{c}
e+d-r-k \\
d-k
\end{array}\right] .
\end{aligned}
$$

Hence, replacing $e$ by $e+d-r$, identity (1.5) can be rephrased as follows:

$$
\sum_{w \in M} q^{\operatorname{inv}(w)}=\sum_{w \in N} q^{\operatorname{inv}(w)}
$$

We now give a bijection $\eta: M \longrightarrow N$ to interpret the above identity. Start with a quadruple $w=\left(w_{1}, w_{2}, w_{3}, w_{4}\right)$ in $M_{k}$. Replacing all the $b-k 1$ 's in $w_{2}$ by the rightmost $b-k$ letters in $w_{1}$, we obtain a word $w_{2}^{\prime}$. Denote by $w_{2}^{\prime \prime}$ the word obtained from $w_{2}^{\prime}$ by deleting all the 1's. Let $v_{1}^{\prime}$ be the word obtained from $w_{2}^{\prime}$ by replacing every 3 by 2 . Note that $w_{2}^{\prime \prime}$ is a word on $\{2,3\}$. Applying Lemma 2.1, we obtain $\left(v_{3}^{\prime}, v_{3}, v_{4}\right)=\theta\left(w_{2}^{\prime \prime}, w_{3}, w_{4}\right)$.

Let $w_{1}^{\prime}$ be the subword of $w_{1}$ corresponding to the leftmost $a+c$ letters. It is easy to see that the number of 1 's in $w_{1}^{\prime}$ is exactly equal to the length of $v_{3}^{\prime}$. Let $w_{1}^{\prime \prime}=\imath_{12}\left(w_{1}^{\prime}\right)$. Replacing all the 2's in $w_{1}^{\prime \prime}$ by the word $v_{3}^{\prime}$, we obtain a word $w_{1}^{\prime \prime \prime}$. Let $v_{2}$ be the word obtained from $w_{1}^{\prime \prime \prime}$ by replacing every 2 by 1 , and let $v_{2}^{\prime}$ be the subword of $w_{1}^{\prime \prime \prime}$ by deleting all the 3's. Finally, let $v_{1}=\imath_{12}\left(v_{1}^{\prime}\right) v_{2}^{\prime}$. Suppose $v_{3}$ has $d-k^{\prime} 3$ 's. Then it is easy to see that $v=\left(v_{1}, v_{2}, v_{3}, v_{4}\right) \in N_{k^{\prime}}$.

Conversely, if $v=\left(v_{1}, v_{2}, v_{3}, v_{4}\right) \in N_{k}$, then the above procedure with $b-k$ changed to $a-k$, and $a+c$ changed to $b+d$ also defines a mapping from $N$ to $M$, also denoted by $\eta$. It is easy to check that $\eta^{2}$ is the identity mapping. Namely, $\eta: w \mapsto v$ is a bijection from $M$ to $N$. Moreover, an argument similar to that in the proof of Lemma 2.1 shows that $\operatorname{inv}(w)=\operatorname{inv}(v)$. This completes the proof.

\section{A Combinatorial Proof of Equation (1.8)}

Replacing $q$ by $q^{2}, y$ by $-y q$, and $z$ by $-z q$, we can rewrite (1.8) as

$$
\sum_{k=0}^{n}(-1)^{k} \frac{\left(-y q ; q^{2}\right)_{k}\left(-z q ; q^{2}\right)_{n-k}}{\left(q^{2} ; q^{2}\right)_{k}\left(q^{2} ; q^{2}\right)_{n-k}} q^{(2 m+1) k} z^{k}=\sum_{k=0}^{\min \{m, n\}}\left[\begin{array}{c}
m \\
k
\end{array}\right]_{q^{2}} \frac{\left(y z q^{2 m+2} ; q^{2}\right)_{n-k} q^{k^{2}} z^{k}}{\left(q^{2} ; q^{2}\right)_{n-k}}
$$

A partition $\lambda$ is a finite sequence of nonnegative integers $\left(\lambda_{1}, \lambda_{2}, \ldots, \lambda_{m}\right)$ such that $\lambda_{1} \geq \lambda_{2} \geq \cdots \geq \lambda_{m} \geq 0$. Each $\lambda_{i}>0$ is called a part of $\lambda$. The numbers of parts, odd parts, and even parts of $\lambda$ are denoted by $\ell(\lambda) \operatorname{odd}(\lambda)$, and even $(\lambda)$, respectively. Write 
$|\lambda|=\sum_{i=1}^{m} \lambda_{i}$, called the weight of $\lambda$. The set of all partitions into even parts is denoted by $\mathcal{P}_{\text {even }}$. The set of all partitions into distinct odd (resp. even) parts is denoted by $\mathcal{D}_{\text {odd }}$ (resp. $\mathcal{D}_{\text {even }}$ ). Let $\mathcal{P}_{1}$ (resp. $\mathcal{P}_{2}$ ) denote the set of partitions with no repeated odd (resp. even) parts. Given two partitions $\lambda$ and $\mu$, we define $\lambda \cup \mu$ to be the partition whose parts are those of $\lambda$ and $\mu$ in decreasing order, and $\lambda+\mu$ to be the partition of which the $i$-th part is the sum of $\lambda_{i}$ and $\mu_{i}$. If $t$ is a part of $\lambda$, then $\lambda \backslash t$ denotes the partition obtained from $\lambda$ by deleting one part equal to $t$.

The following lemma is a combinatorial version of the $q$-binomial theorem, as shown in [7]. See also Chapman [4]. For the convenience of the reader, we sketch a proof here. Other models, as overpartitions, of the $q$-binomial theorem have been given by Joichi and Stanton [9] and Alladi [1]. See also Pak's survey [10].

Lemma 3.1 There is an involution $\sigma$ on $\mathcal{P}_{1}$ such that for each $\lambda \in \mathcal{P}_{1}$, we have

$$
|\sigma(\lambda)|=|\lambda|, \ell(\sigma(\lambda))=\left\lceil\lambda_{1} / 2\right\rceil, \text { and } \operatorname{odd}(\sigma(\lambda))=\operatorname{odd}(\lambda) .
$$

Proof. Given a partition $\lambda \in \mathcal{P}_{1}$, we draw the 2-modular diagram of $\lambda$ as follows: an even part $2 k$ will give a row of $k 2$ 's, while an odd part $2 k+1$ will give a row of $k 2$ 's followed by a 1 . So each part $\lambda_{i}$ corresponds to a row of length $\left\lceil\lambda_{i} / 2\right\rceil$, and the number of 1 's in the 2-modular diagram is odd $(\lambda)$. Since no odd part of $\lambda$ is repeated, the 1's can only occur at the bottom of columns. We identify elements of $\mathcal{P}_{1}$ with their diagrams, and then define $\sigma$ to be conjugation of diagrams. Clearly, the number of rows in the diagram is $\ell(\lambda)$, while the number of columns is $\left\lceil\lambda_{1} / 2\right\rceil$. Thus, $\sigma$ has the required property and Lemma 3.1 is proved.

Example 3.1 Let $\lambda=(10,9,7,4,4,4,3,2,2,1)$. Then, $\lambda$ gives the left 2-modular diagram below, while its conjugation $\sigma(\lambda)$ gives the right 2-modular diagram below:

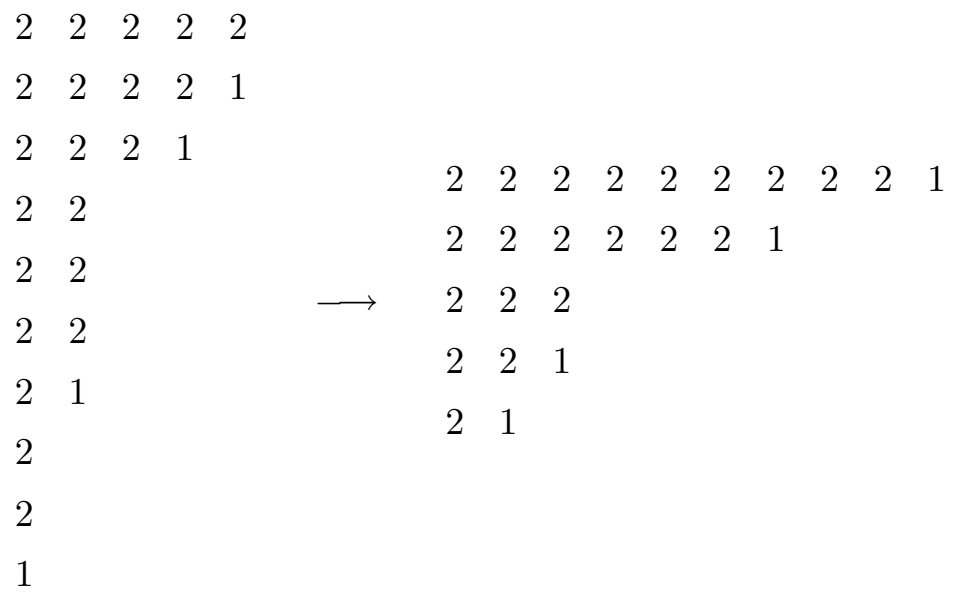

Namely, $\sigma(\lambda)=(19,13,6,5,3)$.

We derive immediately the following result. 
Lemma 3.2 We have

$$
\sum_{\substack{\mu \in \mathcal{P}_{1} \\ \ell(\mu) \leq n}} q^{|\mu|} z^{\operatorname{odd}(\mu)}=\sum_{\substack{\lambda \in \mathcal{P}_{1} \\ \lambda_{1} \leq 2 n}} q^{|\lambda|} z^{\operatorname{odd}(\lambda)}=\frac{\left(-z q ; q^{2}\right)_{n}}{\left(q^{2} ; q^{2}\right)_{n}} .
$$

We also need some other lemmas. Set

$$
\mathcal{A}_{m, n}=\left\{(\lambda, \mu) \in \mathcal{P}_{2} \times \mathcal{P}_{1}: \ell(\lambda)+\ell(\mu) \leq n \text { and } \lambda_{\ell(\lambda)} \geq 2 m+1\right\}
$$

Lemma 3.3 For $m \geq 0$ and $n \geq 1$, we have

$$
\begin{aligned}
\sum_{k=0}^{n} & (-1)^{k} \frac{\left(-y q ; q^{2}\right)_{k}\left(-z q ; q^{2}\right)_{n-k}}{\left(q^{2} ; q^{2}\right)_{k}\left(q^{2} ; q^{2}\right)_{n-k}} q^{(2 m+1) k} z^{k} \\
= & \sum_{(\lambda, \mu) \in \mathcal{A}_{m, n}}(-1)^{\ell(\lambda)} q^{|\lambda|+|\mu|} y^{\operatorname{even}(\lambda)} z^{\operatorname{odd}(\mu)+\ell(\lambda)} .
\end{aligned}
$$

Proof. Let $\nu=(2 m+1, \ldots, 2 m+1)$ be a partition with $k$ parts. By Lemma 3.2,

$$
\frac{\left(-y q ; q^{2}\right)_{k}}{\left(q^{2} ; q^{2}\right)_{k}} q^{(2 m+1) k} z^{k}=q^{|\nu|} z^{\ell(\nu)} \sum_{\substack{\tau \in \mathcal{P}_{1} \\ \ell(\tau) \leq k}} q^{|\tau|} y^{\operatorname{odd}(\tau)}=\sum_{\substack{\lambda \in \mathcal{P}_{2} \\ \ell(\lambda)=k \\ \lambda_{\ell(\lambda) \geq 2 m+1} \geq 2 m+1}} q^{|\lambda|} y^{\operatorname{even}(\lambda)} z^{\ell(\lambda)}
$$

where $\lambda=\tau+\nu$. Also,

$$
\frac{\left(-z q ; q^{2}\right)_{n-k}}{\left(q^{2} ; q^{2}\right)_{n-k}}=\sum_{\substack{\mu \in \mathcal{P}_{1} \\ \ell(\mu) \leq n-k}} q^{|\mu|} z^{\operatorname{odd}(\mu)}
$$

Multiplying the above two identities and summing over $k$, we get the desired identity.

Let $\mathcal{B}_{m, n}$ be the subset of $\mathcal{A}_{m, n}$ consisting of the pairs $(\lambda, \mu)$ such that $\lambda_{i}$ is odd for some $i$, or $\mu_{j}$ is odd for some $j$ and $\mu_{j} \geq 2 m+1$.

Lemma 3.4 For $m \geq 0$ and $n \geq 1$, we have

$$
\sum_{(\lambda, \mu) \in \mathcal{B}_{m, n}}(-1)^{\ell(\lambda)} q^{|\lambda|+|\mu|} y^{\operatorname{even}(\lambda)} z^{\operatorname{odd}(\mu)+\ell(\lambda)}=0 .
$$

Proof. We will construct a weight preserving and sign reversing involution $\phi$ on $\mathcal{B}_{m, n}$. For any $(\lambda, \mu) \in \mathcal{B}_{m, n}$, as no odd part of $\mu$ is repeated, let $t$ be the largest odd part in $\lambda \cup \mu$. By the definition of $\mathcal{B}_{m, n}$, we see that $t \geq 2 m+1$. Now define

$$
\phi((\lambda, \mu))= \begin{cases}(\lambda \cup t, \mu \backslash t), & \text { if } t \text { is a part of } \mu, \\ (\lambda \backslash t, \mu \cup t), & \text { if } t \text { is not a part of } \mu .\end{cases}
$$


It is straightforward to verify that $\phi$ is an involution on $\mathcal{B}_{m, n}$ which preserves $|\lambda|+|\mu|$, even $(\lambda)$ and $\operatorname{odd}(\mu)+\ell(\lambda)$ and reverses the sign $(-1)^{\ell(\lambda)}$.

Proof of (3.1). Note that $(\lambda, \mu) \in \mathcal{A}_{m, n} \backslash \mathcal{B}_{m, n}$ if and only if $\lambda \in \mathcal{D}_{\text {even }}$ and for any $i$ if $\mu_{i}$ is odd then $\mu_{i} \leq 2 m-1$. Combining Lemmas 3.3 and 3.4, we see that the left-hand side of $(3.1)$ is equal to

$$
\begin{aligned}
& \sum_{(\lambda, \mu) \in \mathcal{A}_{m, n} \backslash \mathcal{B}_{m, n}}(-1)^{\ell(\lambda)} q^{|\lambda|+|\mu|} y^{\ell(\lambda)} z^{\text {odd }(\mu)+\ell(\lambda)} \\
= & \sum_{k=0}^{\min \{m, n\}} z^{k} \sum_{\substack{\eta \in \mathcal{D}_{\text {odd }} \\
\ell(\eta)=k \\
\eta_{1} \leq 2 m-1}} q^{|\eta|} \sum_{\substack{\tau \in \mathcal{D}_{\text {odd }} \\
\nu \in \mathcal{P}_{\text {even }} \\
\ell(\tau)+\ell(\nu) \leq n-k}} q^{|\tau|+|\nu|}\left(-y z q^{2 m+1}\right)^{\ell(\tau)},
\end{aligned}
$$

where $k=\operatorname{odd}(\mu), \mu=\eta \cup \nu$, and $\tau_{i}=\lambda_{i}-(2 m+1)$.

Now, setting $\pi_{i}=\eta_{i}-(2 i-1), 1 \leq i \leq k$, and using the result (see [2, Theorem 3.1])

$$
\sum_{\substack{\ell(\alpha) \leq k \\
\alpha 1 \leq m-k}} q^{|\alpha|}=\left[\begin{array}{c}
m \\
k
\end{array}\right]
$$

we have

$$
\sum_{\substack{\eta \in \mathcal{D}_{\text {odd }} \\
\ell(\eta)=k \\
\eta_{1} \leq 2 m-1}} q^{|\eta|}=q^{k^{2}} \sum_{\substack{\pi \in \mathcal{P}_{\text {even }} \\
\ell(\pi) \leq k \\
\pi_{1} \leq 2 m-2 k}} q^{|\pi|}=q^{k^{2}}\left[\begin{array}{c}
m \\
k
\end{array}\right]_{q^{2}} .
$$

Also, replacing $z$ by $-y z q^{2 m+1}$ and $n$ by $n-k$ in (3.2) yields

$$
\sum_{\substack{\tau \in \mathcal{D}_{\text {odd }} \\ \nu \in \mathcal{P}_{\text {even }} \\ \ell(\tau)+\ell(\mu) \leq n-k}} q^{|\tau|+|\nu|}\left(-y z q^{2 m+1}\right)^{\ell(\tau)}=\frac{\left(y z q^{2 m+2} ; q^{2}\right)_{n-k}}{\left(q^{2} ; q^{2}\right)_{n-k}} .
$$

Finally, combining (3.5), (3.6) and (3.7) completes the proof.

\section{An Elementary Proof of Equation (1.3)}

Lemma 4.1 For $m, n \in \mathbb{N}$, we have

$$
\frac{(x q, y q ; q)_{m}}{(q, x y q ; q)_{m}} \sum_{k=0}^{n} \frac{\left(x, y, v q^{m} ; q\right)_{k}}{\left(q, v, x y q^{m+1} ; q\right)_{k}} q^{k}=\frac{(x q, y q ; q)_{n}}{(q, x y q ; q)_{n}} \sum_{k=0}^{m} \frac{\left(x, y, v q^{n} ; q\right)_{k}}{\left(q, v, x y q^{n+1} ; q\right)_{k}} q^{k} .
$$

Proof. For $k \geq 0$, let $B(-1, k)=0$ and

$$
B(r, k)=\frac{(x q, y q ; q)_{r}}{(q, x y q ; q)_{r}} \frac{\left(x, y, v q^{r} ; q\right)_{k}}{\left(q, v, x y q^{r+1} ; q\right)_{k}} q^{k}, \quad r \geq 0 .
$$


For $r, k \geq 0$, set

$$
A(r, k):=B(r, k)-B(r-1, k) .
$$

Then (4.1) may be written as

$$
\sum_{k=0}^{n} \sum_{r=0}^{m} A(r, k)=\sum_{k=0}^{m} \sum_{r=0}^{n} A(r, k)
$$

Since $A(r, k)=A(k, r)$, the above identity is then obvious.

Proof of (1.3). Since both sides of (1.3) are rational fractions of $z$, it suffices to show that (1.3) holds for all $z=q^{c}(c \geq 1)$. We proceed by induction on $c$. The $z=q$ case of (1.3) is equivalent to (4.1) and has been proved. Suppose (1.3) holds for $z=q^{c}$. Denote the left-hand side of (1.3) by $S(m, n, x, z)$ for nonnegative integers $m$ and $n$. Then (1.3) means nothing else that $S(m, n, x, z)$ is symmetric in $m$ and $n$. Multiplying both sides of

$$
\frac{1-x y z^{2} q^{m+n}}{1-x z q^{n}}\left(1-x q^{k}\right)+\frac{x\left(1-y z q^{m}\right)}{1-x z q^{n}}\left(q^{k}-z q^{n}\right)=1-x y z q^{m+k}
$$

by

$$
\frac{1}{1-x y z q^{m+k}} \frac{(x z, y z ; q)_{m}}{(q, x y z ; q)_{m}} \frac{\left(x, y, v q^{m} ; q\right)_{k}(z ; q)_{n-k}}{\left(q, v, x y z q^{m} ; q\right)_{k}(q ; q)_{n-k}} z^{k}
$$

and summing over $k$ from 0 to $n$, we obtain

$$
a S(m, n, x q, z)+b S(m, n, x, z q)=S(m, n, x, z),
$$

where the coefficients $a$ and $b$ are two symmetric expressions in $m$ and $n$ :

$$
a=\frac{\left(1-x y z^{2} q^{m+n}\right)(1-x)(1-x z)}{(1-x y z)\left(1-x z q^{m}\right)\left(1-x z q^{n}\right)}, \quad b=\frac{x(1-z)(1-x z)(1-y z)}{(1-x y z)\left(1-x z q^{m}\right)\left(1-x z q^{n}\right)} .
$$

Since both $S(m, n, x q, z)$ and $S(m, n, x, z)$ are symmetric in $m$ and $n$ by induction hypothesis, we deduce that $S(m, n, x, z q)$ is symmetric in $m$ and $n$, i.e., (1.3) holds for $z q$. This completes the proof.

Remark. Equation (4.2) can be obtained from the $n=1$ case of the $q$-Pfaff-Saalschütz identity [5, (1.7.2)]. Krattenthaler has indicated how to derive contiguous relations as (4.3) from special cases of terminating basic hypergeometric summation or transformation formulas (see "A systematic list of two- and three-term contiguous relations for basic hypergeometric series," available at http://euler.univ-lyon1.fr/home/kratt/papers.html).

Acknowledgments. This work was done under the auspices of the National Science Foundation of China. The second author was supported by EC's IHRP Programme, within Research Training Network "Algebraic Combinatorics in Europe," grant HPRNCT-2001-00272. 


\section{References}

[1] K. Alladi, A fundamental invariant in the theory of partitions, In: Topics in Number Theory (University Park, PA, 1997), Kluwer Academic Publishers, Dordrecht, 1999, pp. 101-113.

[2] G. E. Andrews, The Theory of Partitions, Cambridge University Press, Cambridge, 1998.

[3] G. E. Andrews and D. M. Bressoud, Identities in combinatorics III: further aspects of ordered set sorting, Discrete Math. 49 (1984), 223-236.

[4] R. Chapman, Combinatorial proofs of $q$-series identities, J. Combin. Theory, Ser. A 99 (2002), 1-16.

[5] G. Gasper and M. Rahman, Basic Hypergeometric Series, Encyclopedia of Mathematics and Its Applications, Vol. 96, Second Edition, Cambridge University Press, Cambridge, 2004.

[6] I. P. Goulden, A bijective proof of the $q$-Saalschütz theorem, Discrete Math. 57 (1985), $39-44$.

[7] V. J. W. Guo, The $q$-binomial theorem and two symmetric $q$-identities, Eletron. J. Combin. 10 (2003), \#R34.

[8] V. J. W. Guo, Elementary proofs of some $q$-identities of Jackson and Andrews-Jain, preprint.

[9] J. T. Joichi and D. Stanton, Bijective proofs of basic hypergeometric series identities, Pacific J. Math. 127 (1987), 103-120.

[10] I. Pak, Partition bijections, a survey, Ramanujan J., to appear.

[11] J. Zeng, Sur une formule de Ramanujan-Bailey, Canad. J. Math. 42 (1990), 607-618.

[12] D. Zeilberger, A q-Foata proof of the q-Saalschütz identity, European J. Combin. 8 (1987), 461-463. 